\title{
FlowLab: Computational Fluid Dynamics (CFD) Framework for Undergraduate Education
}

\author{
Richard D. LaRoche, Barbara J. Hutchings, and R. Muralikrishnan \\ Fluent Inc., Lebanon, NH 03766 USA
}

Today, the use of Computational Fluid Dynamics (CFD) software in academia occurs primarily in the context of student projects or research. The potential of CFD as a tool to enhance teaching is largely untapped, despite growing interest in computer tools to assist learning. FlowLab (http://flowlab.fluent.com) is a CFD-based educational software package that will allow students to solve fluid dynamics problems without the long learning curve required by today's commercial CFD packages.

FlowLab provides students with a "Virtual Fluids Laboratory" which would use CFD to teach and visually reinforce concepts in fluid flow and heat transfer. FlowLab introduces students to the effective use of CFD for solving fluid flow problems, providing them with exposure to software tools that are increasingly important in industry. FlowLab allows students to get started immediately without having to spend the large time commitment to learn geometry and mesh creation skills that traditional CFD software require. It also allows lecturers and teachers to easily deploy CFD in undergraduate curricula using pre-defined examples and exercises, with the ability for the teacher to customize the exercises or create their own to tie directly into the existing curriculum. Fluent is working with university professors worldwide to develop a library of FlowLab exercises which would be available freely through the Internet. Below are the overall educational goals for the FlowLab framework:

- Reinforce basic concepts of fluid mechanics and heat/mass transfer using computer simulation

- Use computing exercises to augment and complement existing laboratory-based curriculum

- Expand the learning experience with real-world applications of fluid flow and heat/mass transfer

- Expose students to CFD and CFD concepts - an increasingly important skill in the job market

\section{Customizing FlowLab}

FlowLab is designed to fit easily into existing curriculum by using the idea of customized exercises. Exercises consist of two parts. First, a parameterized template defines the inputs that drive the simulation. Those inputs can be as simple or as complex as the exercise authors desire, and are determined when the parameterized template is created. Secondly, each template is accompanied by exercise notes. The exercise notes tell the student what simulation tasks to perform and guide the student's interpretation of results.

\footnotetext{
"Proceedings of the 2002 American Society for Engineering Education Annual Conference \& Exposition Copyright (C) 2002, American Society for Engineering Education”
} 
Customizing FlowLab exercises can be done in the following ways:

- Use existing templates and exercise notes

- Customize the exercise notes

- Create a new template

Customizing Exercise Notes

The exercise notes are electronic and customizable by the teacher. They can be done in any format that is convenient to the teacher and students (html, MSWord, pdf or in a FlowLab window). These notes lead the student through the exercise, focusing on reinforcing basic fluid mechanics concepts through specific post-processing and/or 'what-if' studies.

Creating New Templates

The instructor can create new FlowLab exercises using Fluent's standard CFD software (FLUENT and GAMBIT). The expertise required to create new FlowLab templates is not too much more than what is needed to setup and test a new FLUENT problem. Many professors have used FLUENT in the past for senior projects or independent study. These projects would be an ideal opportunity to create a new FlowLab exercise which can be used in future undergraduate classes.

We are collaborating with university professors all over the world to create new FlowLab exercises. This ever-growing library of templates will be available to all FlowLab users through our website at http://flowlab.fluent.com. Below are descriptions of a few FlowLab exercises designed for introductory fluid mechanics courses.

\section{Sudden Expansion in a Pipe (axisymmetric, incompressible, turbulent)}

This exercise allows students to model incompressible, turbulent flow in a sudden pipe expansion. Students can prescribe inlet diameter, inlet section length, outlet diameter, fluid velocity, viscosity and density. After selecting a mesh size and solving, students use the CFD results to examine static vs. total pressure and to perform an integral momentum balance and explain any losses. They can verify the average velocity predicted at the exit via a simple mass balance. Students can create color contour and vector plots of the CFD solution. The following $\mathrm{X}-\mathrm{Y}$ plots are predefined where students can input experimental data or literature values for comparison to the CFD results:

- Centerline Velocity

- Centerline Pressure

- Centerline Total Pressure

- Exit Pipe Wall Pressure

- Exit Pipe Wall Total Pressure

- Exit Pipe Wall Total Pressure

"Proceedings of the 2002 American Society for Engineering Education Annual Conference \& Exposition Copyright $\odot$ 2002, American Society for Engineering Education” 


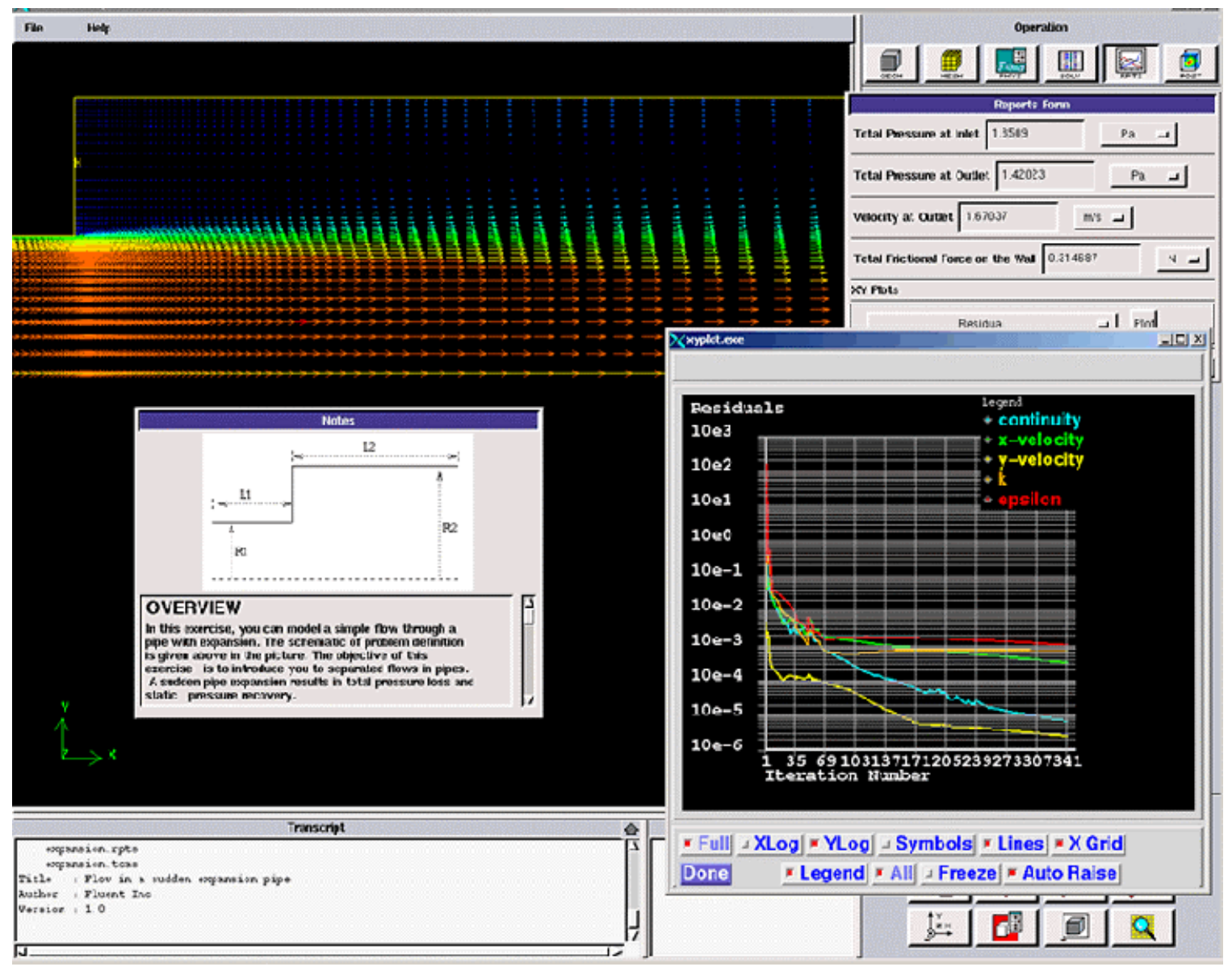

Figure 1. Fluent/FlowLab showing the velocity vectors and convergence history of a sudden pipe expansion.

\section{Developing Flow and Heat Transfer in a Pipe (axisymmetric, incompressible, laminar/turbulent)}

This exercise allows students to read the parameterized geometry and select fluid properties and a pipe diameter to achieve a specified Reynolds number. Students input the pipe length and appropriate boundary conditions to examine developing flow in a pipe. Students can choose different mesh densities to study impact of mesh dependence on their laminar flow solution. Exercise notes guide the students in examining the developing velocity profiles and comparing the fully developed profile to the Blasius solution. Pressure drop in the entrance length and fully developed sections should be plotted. Wall shear stress could be examined/computed and an overall momentum balance could be performed to equate wall shear to the pressure loss. The entrance length to fully-developed flow can be compared to the expected value for the selected Reynolds number. Heat transfer can be added with students selecting properties to achieve a specified Prandtl number. Students can create color contour and vector plots of the CFD solution.

"Proceedings of the 2002 American Society for Engineering Education Annual Conference \& Exposition Copyright (C) 2002, American Society for Engineering Education” 
The following X-Y plots are predefined where students can input experimental data or literature values for comparison to the CFD results:

- Centerline Velocity

- Centerline Pressure

- Centerline Temperature

- Wall Friction Factor

- Wall Temperature

- Wall Nusselt Number

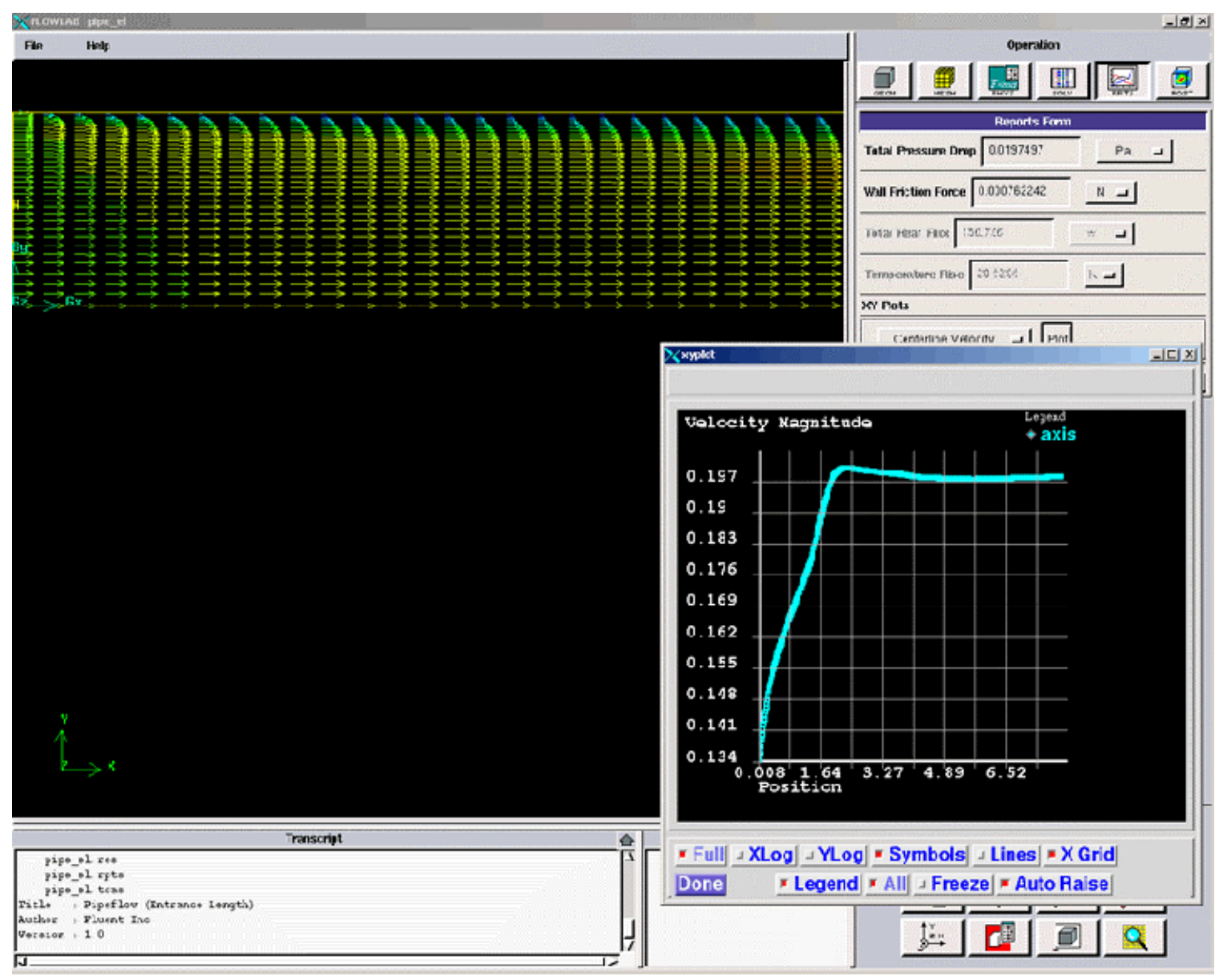

Figure 2. Fluent/FlowLab showing Developing Pipe Flow at Re=2000.

\section{Flow Around a Cylinder (2D, incompressible, laminar)}

In this exercise, students set cylinder diameter, mesh density and whether they want an inviscid or viscous solution. They set velocity and properties to achieve a particular Reynolds number (Re). Students can perform computational experiments at varying $\mathrm{Re}$ so they can generate a $\mathrm{Re}$ vs. Cd plot. Students can create color contour and vector plots of the CFD solution. The 
following X-Y plots are predefined where students can input experimental data or literature values for comparison to the CFD results:

- Pressure Coefficient Distribution

- Pressure Distribution

- Friction Coefficient

- X-Wall Shear Stress Distribution

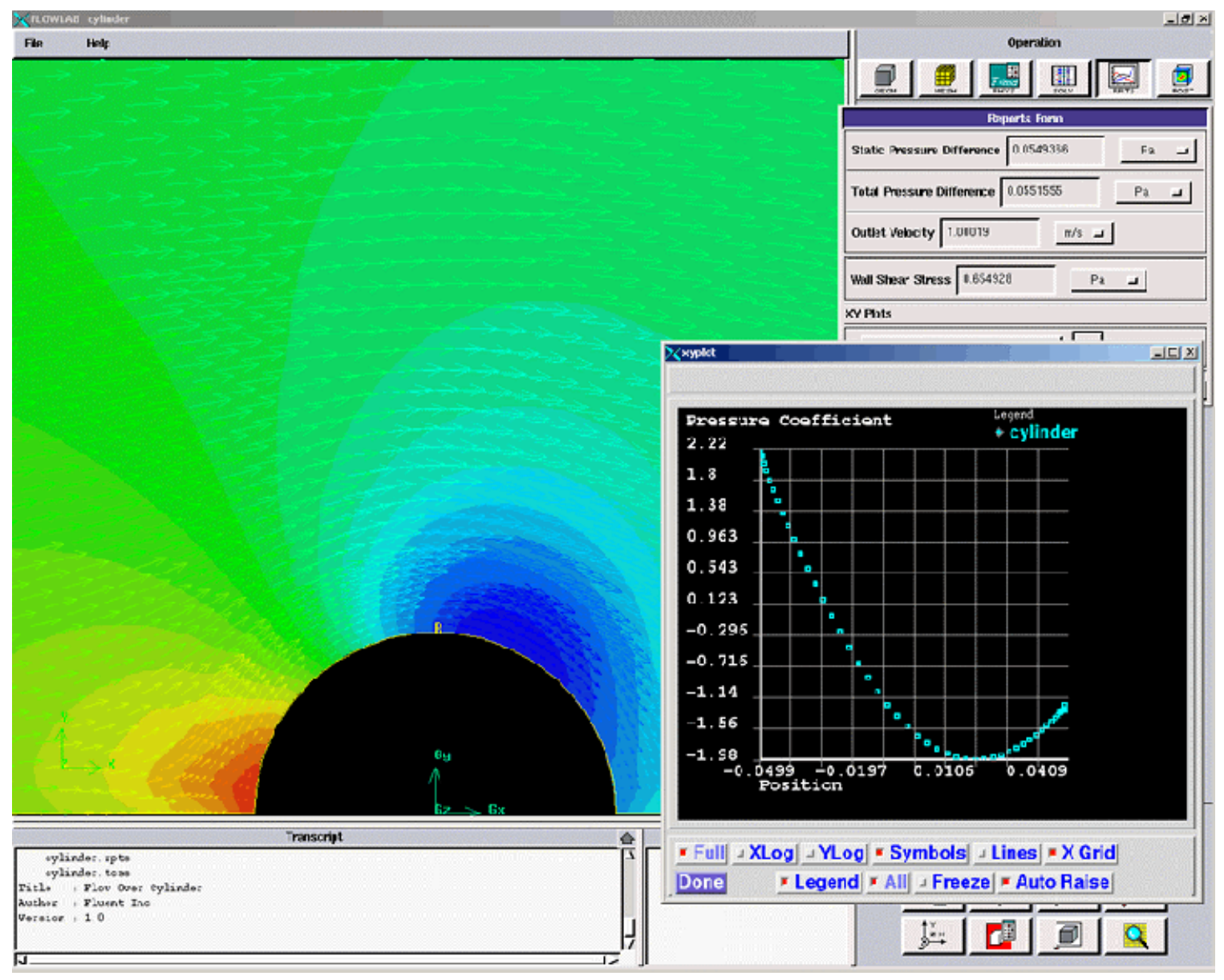

Figure 3. Fluent/FlowLab showing pressure contours, velocity vectors and pressure coefficient distribution for flow past a cylinder at Re=10.

\section{Lift/Drag on an Airfoil (2D, compressible, turbulent)}

In this exercise, the students study the flow around a 2D Clark-Y airfoil. They have the option of choosing invicid or viscous flow along with different mesh densities. Input parameters include far field temperature \& pressure, free stream velocity and angle of attack. The students will be able to explore how these parameters affect wall shear stress, skin friction factor, far field 
pressure coefficient, lift coefficient and drag coefficient. Students can create color contour and vector plots of the CFD solution. The following X-Y plots are predefined where students can input experimental data or literature values for comparison to the CFD results:

- Pressure Coefficient

- Friction Coefficient

- Shear Stress Distribution

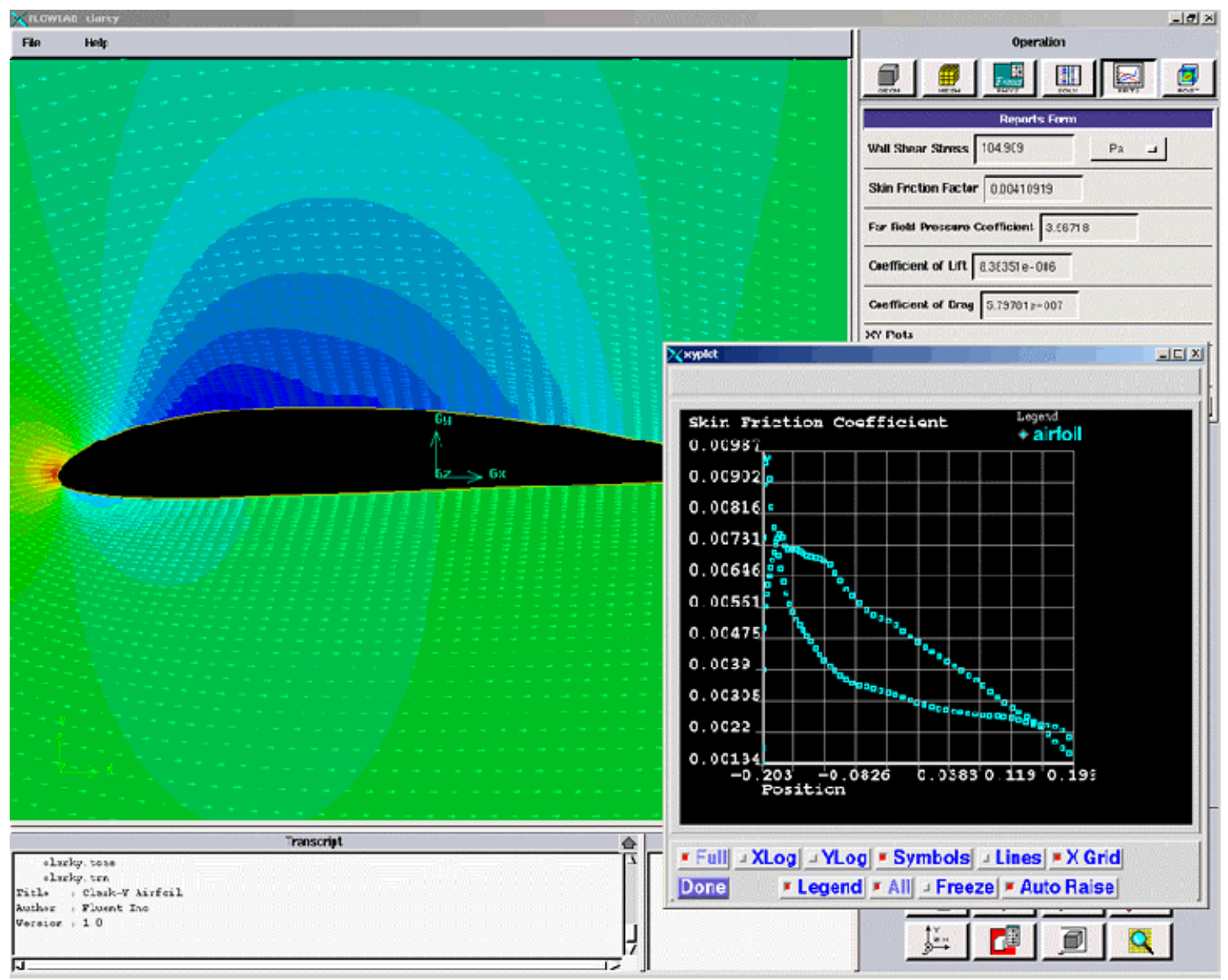

Figure 4. Fluent/FlowLab showing pressure contours, velocity vectors, and skin friction coefficient of a Clark-Y Airfoil at Mach 0.6

\section{Converging/Diverging Nozzle (axisymmetric, compressible, transonic/supersonic flow)}

Students define the following geometric parameters: inlet radius, throat radius, outlet radius, converging length, and diverging length. They can set the upstream stagnation pressure/temperature and run successive simulations with varying back pressure. Students would be able to check for choked flow by performing mass flow rate integrations. They could display shocks and compare the pressure and Mach number to isentropic relationships. Output results include Mach Number and static pressure at the inlet, outlet and throat. Students can create color 
contour and vector plots of the CFD solution. The following X-Y plots are predefined where students can input experimental data or literature values for comparison to the CFD results:

- Wall Pressure

- Wall Mach Number

- Centerline Pressure

- Centerline Mach Number

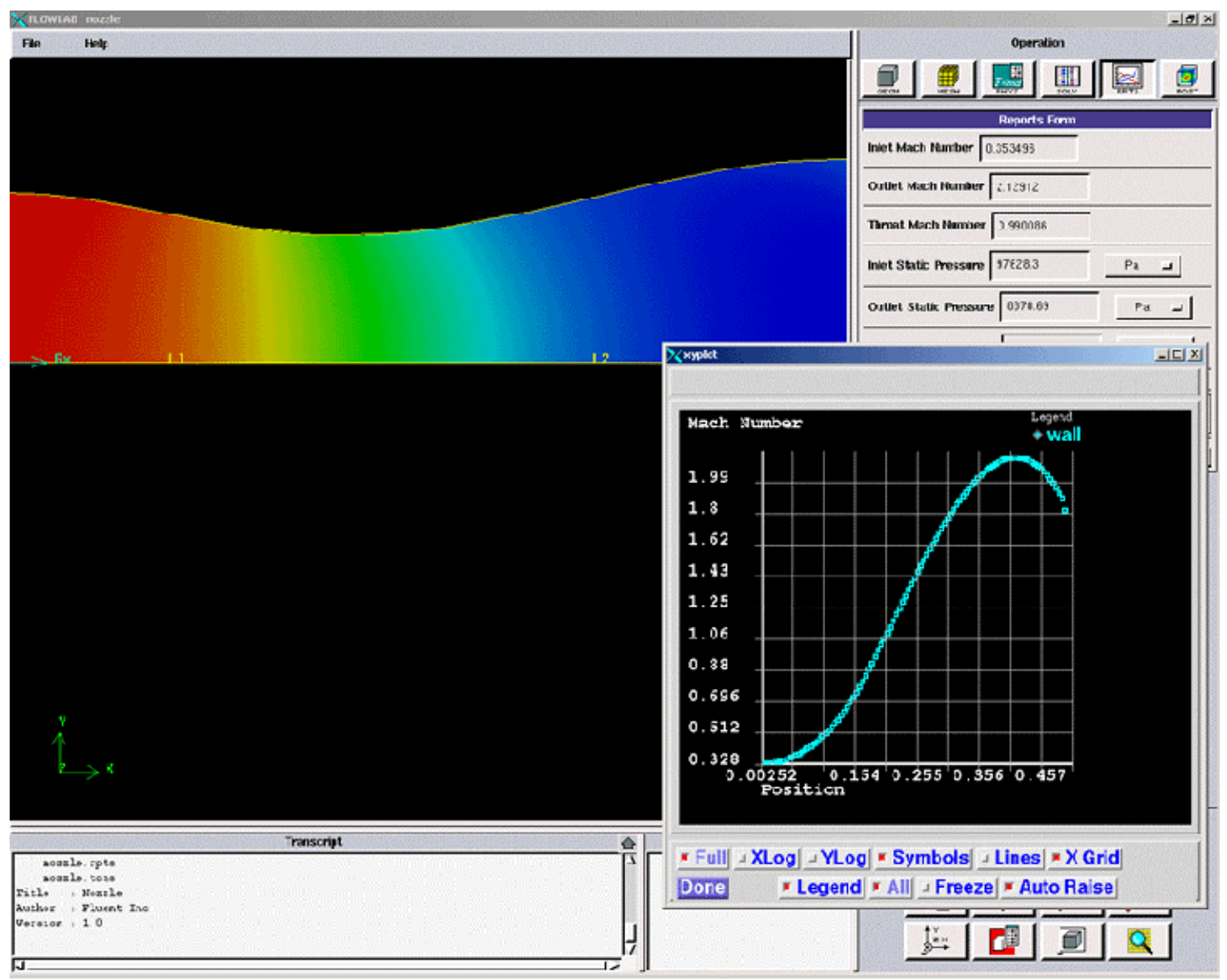

Figure 5. Fluent/Flowlab Showing Pressure Contours and Wall Mach Number for a Converging/Diverging Nozzle

\section{University Collaboration}

At the time of this writing, FlowLab exercises are being evaluated and developed at enginering departments (aerospace, chemical, civil, mechanical) at over 30 universities worldwide. Our academic partners are working with us to develop university collaboration and peer-review procedures in the development of FlowLab exercises for engineering classes in fluid dynamics and heat transfer. 


\section{Acknowledgments}

The authors are grateful for the feedback from the over 30 universities involved in the FlowLab beta testing program. We acknowledge the work of Apurva Shukla who while at Fluent India worked on FlowLab template development. Finally, we thank the FlowLab development team (Harsh Vardan, Frank Li, Nilanjan Sasmal, Suryakiran Gullapalli) for their work in developing this framework.

In the spirit of disseminating information about the FlowLab framework to the broader academic engineering community, portions of this paper will also be presented at the 2002 American Society of Mechanical Engineers (ASME) Fluids Engineering Division Summer Meeting to be held in Montreal, Quebec, Canada, July 14-18, 2002.

RICHARD D. LAROCHE joined Fluent Inc. in 2001 as U.S. University Program Manager and FlowLab Product Manager after over 17 years of engineering work experience in both academia and industry. Prior to joining Fluent, Richard was a fluid flow consultant for DuPont Engineering. He received his B.S. degree in chemical engineering from Montana State University and his M.S. and Ph.D. degrees from the University of Illinois at UrbanaChampaign. Richard is a former professor of Penn State University and a former adjunct faculty member at the University of Delaware. He has also held engineering positions at Cray Research and Dow Chemical.

BARBARA J. HUTCHINGS is currently the Director of Strategic Partnership at Fluent Inc., the leading commercial supplier of Computational Fluid Dynamics (CFD) software. Barbara joined Fluent at its inception in 1983, after graduating from the Thayer School of Engineering at Dartmouth College with an M.S. degree. She has been working in the field of applied CFD for 20 years and has an active interest in the use of software tools for engineering education.

R. MURALIKRISHNAN joined Fluent India in 2001 to be responsible for the development of the FlowLab Exercise Library. Muralikrishnan earned an M.Sc. degree from the Indian Institute of Science in 1999 and a B.Tech. degree from Madurai Kamraj in 1997. He has research experience in experimental fluid mechanics and electrochemical engineering. Before joining Fluent, Muralikrishnan worked at Futurica Petrochemicals on engineering software development projects. 